\title{
Effects of epidural anesthesia and postoperative epidural analgesia on immune function in esophageal carcinoma patients undergoing thoracic surgery
}

\author{
CHENG-YONG GU ${ }^{1}$, JIN ZHANG ${ }^{2}$, YAN-NING QIAN ${ }^{2}$ and QI-FENG TANG ${ }^{3}$ \\ ${ }^{1}$ Department of Anesthesiology, Affiliated Suzhou Hospital of Nanjing Medical University, Suzhou, Jiangsu 215008; \\ ${ }^{2}$ Department of Anesthesiology, First Affiliated Hospital of Nanjing Medical University,Nanjing, Jiangsu 210029; \\ ${ }^{3}$ Department of Anesthesiology, Suzhou BenQ Medical Center, Nanjing Medical University, \\ Suzhou, Jiangsu 215009, P.R. China
}

Received October 3, 2012; Accepted June 24, 2014

DOI: $10.3892 / \mathrm{mco} .2014 .405$

\begin{abstract}
Thoracic epidural anesthesia (TEA) has been demonstrated to significantly reduce stress and immune dysfunction in trauma patients. In esophageal carcinoma patients undergoing thoracic surgery, TEA combined with general anesthesia during surgery and subsequent postoperative patient-controlled epidural analgesia (PCEA) may improve plasma cortisol (Cor), interleukin (IL)-6 and IL-17 levels and helper T-cell differentiation. A total of 60 esophageal carcinoma patients undergoing thoracic surgery were randomly allocated into groups I, II, III and I $(n=15$ per group). During surgery, groups I and II received total intravenous general anesthesia (TIVA), whereas groups III and IV received combined TEA and TIVA. Postoperatively, groups I and III received postoperative patient-controlled intravenous analgesia (PCIA), while groups II and IV received PCEA. The Cor, IL-6, IFN- $\gamma$, IL-4 and IL-17 levels were measured in peripheral blood samples collected prior to anesthesia (T0), at $2 \mathrm{~h}$ after incision (T1), at $4 \mathrm{~h}$ postoperatively (T2), at $24 \mathrm{~h}$ postoperatively (T3) and at $48 \mathrm{~h}$ postoperatively (T4). The plasma Cor, IL-17 and IL-6 levels increased significantly at the beginning of the operation in groups I, II and III, while in group IV there were no significant differences during the entire period, concurrent with enhanced Th0 to Th2 shift, contributing to a Th2-dominant Th1/Th2 ratio. General anesthesia with TEA more efficiently inhibited the onset of the Th2-dominant status and decreased the plasma levels of Cor and IL-6 compared to general anesthesia alone and PCEA inhibited the Th2-dominant status more efficiently compared to PCIA. Therefore, general anesthesia combined with TEA
\end{abstract}

Correspondence to: Dr Qi-Feng Tang, Department of Anesthesiology, Suzhou BenQ Medical Center, Nanjing Medical University, 181 Zhuyuan Road, Suzhou, Jiangsu 215009, P.R. China E-mail: sztqf2001@yahoo.com.cn

Key words: esophageal cancer, esophageal surgery, epidural anesthesia, immune function, epidural analgesia and sole administration of PCEA were demonstrated to inhibit the stress response and minimize immune dysfunction, generating most pronounced results upon combination TEA/PCEA treatment.

\section{Introduction}

In the majority of invasive surgical techniques, including those commonly clinically applied in thoracic surgery, the use of anesthesia during surgery and the use of postoperative analgesia for pain control is commonplace practice. However, several existing techniques have been documented to result in increased morbidity and decreased quality of life, due to major systemic inflammatory reactions, acute immunodepression and recurrent pain. In cases where tissue injury is extensive, often affecting adjacent nerves, the patients may undergo prolonged periods of immunodepression lasting for up to 1 week, significantly increasing the risk of infection and other pathological complications (1). Although there have been significant improvements in the modern techniques for postoperative pain control over the past decades, the prevalence of neuropathic pain conditions that affect sensory processing in damaged tissues often results in failure of pain medications to adequately control pain (2).

Neuropathic pain is defined as the onset of chronic pain in the postoperative period following injury, infection or inflammation of peripheral nerves associated with surgery and it may be associated with immune dysfunctions initially presenting directly after surgery (1). It was previously demonstrated that immune and glial activation play an active role in the etiology and symptomatology of such pathological pain in humans, resulting in increased release of the proinflammatory cytokines tumor necrosis factor (TNF), interleukin (IL)-1 and IL-6 from activated immune and glial cells originating in damaged tissues (2). As the levels of these compounds increase, a unique immune-to-brain communication pattern may be initiated that orchestrates not only the increase in pain, but also a variety of physiological, behavioral and hormonal changes that may exert detrimental long-term effects on patient health (3). These compounds may 
also induce neuronal hyperexcitability, resulting in pain that is not adequately managed by the majority of postoperative drugs that are commonly used in clinical surgical practices.

Interferon (IFN)- $\gamma$ is a T-helper (Th) type 1 (Th1) cytokine recognized for its involvement in increasing the cytotoxic activity of immune T cells and NK cells, associated with a rise in proinflammatory cytokine levels in damaged tissues. The action of IFN- $\gamma$ is counterbalanced by IL-4, a Th2 cytokine reported to increase humoral immunity and suppress the Th1 response (4). Proinflammatory immune action is further exacerbated by IL-17 produced by Th17 cells. These compounds act on a broad range of cell types to induce the expression of various cytokines, including IL-6, IL-8, TNF- $\alpha$ and granulocyte-macrophage colony-stimulating factor. IL-17 may also increase the expression levels of the chemokines CXCl1 and CXCl10, as well as a range of metalloproteinases, thus perpetuating postoperative tissue inflammation $(4,5)$. Traditionally, drugs for pain control do not target the immune response in postoperative tissues, resulting in a failure to eliminate the underlying cause of neuropathic pain responsible for increased morbidity, chronic pain and other postsurgical complications.

The present study is a prospective, randomized trial designed to evaluate the levels of immune-associated compounds found in the plasma of patients following invasive thoracic surgery for the treatment of esophageal carcinoma. Patients undergoing open thoracic surgery, an extremely invasive procedure, received various anesthetic and postoperative analgesic therapies. The levels of compounds previously demonstrated to be predominantly associated with the development of neuropathic pain, including Cor, IL-6, IFN- $\gamma$ (marker for Th1 cells), IL-4 (marker for Th2 cells) and IL-17 (marker for Th17 cells), were measured in order to evaluate the effectiveness of thoracic epidural anesthesia (TEA) and postoperative patient-controlled epidural analgesia (PCEA) in maintening improved immunological function subsequent to invasive surgery. Thus, this study investigated a clinically viable alternative treatment for the reduction of the occurrence of chronic postoperative neuropathic pain.

\section{Materials and methods}

Patients. A total of 60 esophageal carcinoma patients undergoing thoracic surgery, with an American Society of Anesthesiologists (ASA) physical status of I or II, were included in the present study. All the patients underwent invasive open surgery. No subjects undergoing minimally invasive surgery were included. The exclusion criteria included kidney or liver function impairment, moderate or severe anaemia, a history of asthma, blood coagulation disorders of any type, allergy to non-steroidal anti-inflammatory drugs, infection within the previous 2 weeks, peptic ulcer within the previous 6 months, radiotherapy, chemotherapy, immunodepressant drug therapy, blood transfusion prior to or during surgery and long-acting medication of any type capable of affecting immune function. The lead physician independently conducted patient assessments for inclusion based on the patient's medical history. Subsequently, the patients were randomly assigned to groups I, II, III and IV ( $\mathrm{n}=15$ per group) using a computer generated table of random numbers. The lead physician was also primarily responsible for drug preparation in order to ensure consistency of drug administration throughout the study.

The study protocol was approved by the Ethics Committee of the Affiliated Suzhou Hospital of Nanjing Medical University and all the patients provided written informed consent prior to inclusion.

Surgical anesthesia. Postoperative patient-controlled intravenous analgesia (PCIA), PCEA and TEA were used for pain control. Surgical anesthesia was administered by group, with group I receiving general anesthesia with PCIA, group II receiving general anesthesia with PCEA, group III receiving general anesthesia with TEA and PCIA and group IV receiving general anesthesia with TEA and PCEA. A solution of phenobarbital sodium $(0.1 \mathrm{~g})$ and atropine $(0.5 \mathrm{mg})$ was injected intramuscularly $30 \mathrm{~min}$ prior to anesthesia. During the operation, venous access to the median cubital vein was established using an 18-gauge cannula and the patients were continuously monitored using a combination of electrocardiographic, invasive blood pressure recording and pulse oximetric techniques. Prior to induction in groups II, III and IV, an 18-gauge epidural catheter was placed at the vertebral interspace between $\mathrm{T} 7$ and T8 and was advanced 3-5 cm in the cephalad direction, placing it directly into the epidural space. Following epidural infusion of $2 \%$ lidocaine $(3 \mathrm{ml}), 0.25 \%$ ropivacaine was administered at $5-7 \mathrm{ml} / \mathrm{h}$. Identification of the analgesia level (T4-L1) was conducted by pinprick perception in groups III and IV. No other drugs were administered to patients in group II via epidural catheter after epidural infusion of $2 \%$ lidocaine $(3 \mathrm{ml})$. All the patients received intravenous anesthesia induction with $2.5 \mu \mathrm{g} / \mathrm{kg}$ fentanyl (50 $\mu \mathrm{g} / \mathrm{ml}$; Renfu, Hubei, China) and $1.5 \mathrm{mg} / \mathrm{kg}$ propofol (10 mg/ml; AstraZeneca, Basiglio, Italy), followed by $2 \mathrm{mg} / \mathrm{kg}$ succinylcholine. Following endotracheal intubation, continuous infusions of propofol and fentanyl were titrated to maintain a bispectral index (BSI) monitor reading of 40 and 60 in all the groups.

Postoperatively, the patients in groups I and III were administered PCIA. All the patients were connected to a postoperative patient-controlled analgesia administration pumping device (PCA pump) and a balloon infuser $(2 \mathrm{ml} / \mathrm{h}$, Apon Corp., Nantong, China). The PCA pumps were filled with tramadol $(10 \mathrm{mg} / \mathrm{ml})$ in saline solution and set with a bolus of $3 \mathrm{ml}$ (corresponding with the $30 \mathrm{mg}$ tramadol demand dose), a lockout interval of $10 \mathrm{~min}$ and a daily maximum dose of $900 \mathrm{mg}$. The patients in groups II and IV were connected to a PCEA pump filled with fentanyl $2 \mu \mathrm{g} / \mathrm{ml}$ in $250 \mathrm{ml}$ of $0.125 \%$ ropivacaine. Each patient was administered a PCEA solution $(10 \mathrm{ml})$ at the first trigger, followed by $5-\mathrm{ml}$ deliveries. The lockout time was $20 \mathrm{~min}$ in the absence of a 4-h limitation or continuous background infusion.

Blood samples. Peripheral blood samples for the measurement of cortisol (Cor), IL-6, IFN- $\gamma$, IL-4 and IL-17 were collected prior to the induction of anesthesia (T0), at $2 \mathrm{~h}$ after incision (T1), at $4 \mathrm{~h}$ postoperatively (T2), at $24 \mathrm{~h}$ postoperatively (T3) and at $48 \mathrm{~h}$ postoperatively (T4). The sampled blood was collected into EDTA tubes and centrifuged at 1,006.2 x $\mathrm{g}$ for $10 \mathrm{~min}$ at $4^{\circ} \mathrm{C}$ immediately after sampling. Thereafter, the plasma samples were stored at $-70^{\circ} \mathrm{C}$ until all the samples were collected. 
Table I. Clinical and demographic data for patients in each of the four groups.

\begin{tabular}{|c|c|c|c|c|c|c|c|}
\hline Groups & $\begin{array}{c}\text { Patient } \\
\text { no. }\end{array}$ & Age (years) & Weight (kg) & Gender $(\mathrm{M} / \mathrm{F})$ & $\operatorname{ASA}(\mathrm{I} / \mathrm{II})$ & $\begin{array}{c}\text { Operation } \\
\text { duration (min) }\end{array}$ & $\begin{array}{l}\text { Intraoperative } \\
\text { bleeding }(\mathrm{ml})\end{array}$ \\
\hline I & 15 & $58.5 \pm 5.2$ & $58.7 \pm 9.5$ & $13 / 2$ & $2 / 13$ & $164.8 \pm 13.2$ & $250.6 \pm 25.9$ \\
\hline II & 14 & $58.1 \pm 5.3$ & $61.2 \pm 7.1$ & $12 / 2$ & $4 / 10$ & $168.7 \pm 12.1$ & $261.4 \pm 20.7$ \\
\hline III & 14 & $56.2 \pm 5.2$ & $61.9 \pm 9.7$ & $9 / 5$ & $3 / 11$ & $165.2 \pm 15.3$ & $249.5 \pm 30.2$ \\
\hline IV & 14 & $54.9 \pm 5.4$ & $61.0 \pm 6.8$ & $11 / 3$ & $3 / 11$ & $167.6 \pm 14.5$ & $257.8 \pm 28.4$ \\
\hline P-value & & 0.46 & 0.75 & 0.55 & 0.77 & 0.85 & 0.58 \\
\hline
\end{tabular}

Quantitative data are expressed as means \pm SD. Categorical data are described as absolute frequencies. ASA, American Society of Anesthesiologists physical status.

Detection of plasma Cor, IL-6, IL-4, IFN- $\gamma$ and IL-17 levels. Cor and IL-6 levels in the plasma were measured by radioimmunoassay (North Biotechnology Corp., Beijing, China). The levels of IFN- $\gamma$, IL-4 and IL-17 in the plasma were measured using commercial quantitative sandwich enzyme-linked immunosorbent assay kits (Quantikine; R\&D Systems, Minneapolis, MN, USA), according to the manufacturer's instructions. The IFN- $\gamma / \mathrm{IL}-4$ ratio was used to determine the Th1/Th2 cell ratio. Standards were prepared and the appropriate volume of sample or standard was added to a 96-well polystyrene microtiter plate pre-coated with either monoclonal antibodies to the appropriate cytokine or to the related antigen (RA). All samples and standards were run in duplicate and the plates were incubated for the recommended time. Each well was subsequently aspirated and the plates were washed with the provided buffered surfactant. An enzyme-linked polyclonal antibody against the cytokine or RA was then added and the plates were incubated a second time prior to final washing. After addition of the substrate solution to each well, the optical density was measured at the appropriate wavelength for each assay period. All the values were reported as $\mathrm{pg} / \mathrm{ml}$. The intraand interassay coefficients of variation of the immunoassay kits ranged from 5 to $10 \%$. Cross-reactivity with other factors was considered negligible in all the cytokine assays.

Statistical analysis. All quantitative data are expressed as means \pm SD. The patient characteristics, including gender, age, weight, operation time and intraoperative bleeding, were evaluated by one-way analysis of variance (ANOVA). Categorical data were described as absolute frequencies and analyzed using the Pearson's $\chi^{2}$ and Fisher's exact tests. The Cor, IL-6, IL-4, IFN- $\gamma$ and IL-17 levels were constructed using ANOVA with the Fisher's least significant difference t-test for post hoc analysis. $\mathrm{P}<0.05$ was considered to indicate a statistically significant difference. All the data were analyzed using the SPSS statistical software, version 12.0 (SPSS Inc., Chicago, IL, USA).

\section{Results}

Study population. Of the 60 enrolled patients, 3 were excluded from the study due to difficulties during placement of the epidural catheter, whereas the remaining 57 patients completed the study. In those patients that completed the study, no complications were observed during the 48-h experimental period and further follow-up was not conducted. There were no significant differences between the four groups regarding age, weight, gender ratio, ASA physical status, duration of operation or intraoperative bleeding (Table I).

Plasma concentrations of Cor and IL-6. Compared to the levels observed at T0, the levels of Cor in groups I and II at $\mathrm{T} 1$ were significantly increased $(\mathrm{P}<0.05)$; however, these levels in group III began to rise at $\mathrm{T} 3(\mathrm{P}<0.05)$, Similarly, groups II and IV exhibited an earlier return to T0 levels, occurring by $\mathrm{T} 4(\mathrm{P}<0.05)$. The Cor levels in group IV did not exhibit significant differences during the entire period. Compared with group IV, the levels of Cor in group I were higher at T1, $\mathrm{T} 2$, T3 and T4 $(\mathrm{P}<0.05)$. In group II, these levels were higher at T2 $(\mathrm{P}<0.05)$, whereas group III exhibited higher levels at $\mathrm{T} 3$ and $\mathrm{T} 4(\mathrm{P}<0.05)$. Compared to group III, the Cor levels in group I were higher compared to those observed at either T1 or T2 $(\mathrm{P}<0.05)$; however, the levels in group II were lower at T4 $(\mathrm{P}<0.05)$ (Table II).

Compared to the levels observed at T0, the levels of IL-6 in group II were significantly increased at $\mathrm{T} 2(\mathrm{P}<0.05)$; in group I, the IL-6 levels exhibited a similar tendency, although the difference was not statistically significant. In group III, the IL-6 levels began to rise at T3 $(\mathrm{P}<0.05)$, whereas in group IV there was no significant difference in IL-6 levels during the entire period. Compared to group IV, the levels of IL-6 in group I were higher at T1, T2 and T4 $(\mathrm{P}<0.05)$. The levels of IL-6 in group II were higher compared to those in group IV at both T1 and T2 $(\mathrm{P}<0.05)$ and these IL-6 levels were higher compared to those observed in group III at T4 $(\mathrm{P}<0.05)$ (Table III).

IFN- $\gamma$ and IL-4 plasma concentrations and IFN- $\gamma /$ IL-4 ratio. Compared to the levels observed at T0, the IFN- $\gamma / \mathrm{IL}-4$ ratio in groups I and II was decreased significantly at T2 $(\mathrm{P}<0.05)$; however, the IFN- $\gamma / \mathrm{IL}-4$ ratio in groups III and IV began to decrease significantly by $\mathrm{T} 3(\mathrm{P}<0.05)$. The IFN- $\gamma / \mathrm{IL}-4$ ratios observed in groups III and IV at T2 were higher compared to those observed in groups I and II $(\mathrm{P}<0.05)$. In addition, the IFN- $\gamma / \mathrm{IL}-4$ ratio in group IV at T3 and T4 was higher compared to that in group I $(\mathrm{P}<0.05)$ (Table IV).

Plasma concentrations of IL-17. The levels of IL-17 in groups I and II at T2 and T3 were higher compared to those 
Table II. Plasma concentrations of cortisol (ng/ml).

\begin{tabular}{lccccccc}
\hline Groups & No. & T0 & T1 & T2 & T3 & T4 & P-value \\
\hline I & 15 & $151.4 \pm 38.8$ & $210.3 \pm 67.0$ & $246.1 \pm 78.8$ & $273.8 \pm 54.8$ & $270.7 \pm 44.6$ & $<0.01$ \\
II & 14 & $160.7 \pm 42.7$ & $201.2 \pm 38.2$ & $222.8 \pm 50.7$ & $238.7 \pm 71.9$ & $188.2 \pm 53.1$ & $<0.01$ \\
III & 14 & $153.6 \pm 36.2$ & $170.4 \pm 60.4$ & $180.7 \pm 55.1$ & $276.3 \pm 89.7$ & $249.3 \pm 59.1$ & $<0.01$ \\
IV & 14 & $173.3 \pm 41.5$ & $166.9 \pm 46.8$ & $172.8 \pm 48.7$ & $216.6 \pm 73.5$ & $181.3 \pm 69.0$ & 0.16 \\
P-value & & 0.46 & $<0.01$ & $<0.01$ & 0.09 & $<0.01$ \\
\hline
\end{tabular}

Differences between groups by time period: T0, no significance. T1, I vs. III, $\mathrm{P}<0.01$; I vs. IV, $\mathrm{P}=0.04$; II vs. III, $\mathrm{P}<0.01 ;$ and III vs. IV, $\mathrm{P}<0.01$. T2, I vs. III, $\mathrm{P}<0.01$; I vs. IV, $\mathrm{P}<0.01$; and II vs. IV, $\mathrm{P}=0.03$. T3, I vs. IV, $\mathrm{P}=0.04$; and III vs. IV, $\mathrm{P}=0.04$. T4, I vs. II, $\mathrm{P}<0.01 ; \mathrm{I}$ vs. IV, $\mathrm{P}<0.01$; II vs. III, $\mathrm{P}<0.01$; and III vs. IV, $\mathrm{P}<0.01$. Differences between time periods compared to T0 for each group: I, T0 vs. T1, $\mathrm{P}<0.01 ; \mathrm{T} 0$ vs. T2, $\mathrm{P}<0.01$; T0 vs. T3, $\mathrm{P}<0.01$; and T0 vs. T4, $\mathrm{P}<0.01$. II, T0 vs. T1, $\mathrm{P}=0.04$; T0 vs. T2, $\mathrm{P}<0.01$; and T0 vs. T3, $\mathrm{P}<0.01$. III, T0 vs. T3, $\mathrm{P}<0.01 ;$ and T0 vs. T4, $\mathrm{P}=0.01$. IV, no significance.

Table III. Plasma concentrations of IL-6 $\left(10^{-3} \mathrm{pg} / \mathrm{ml}\right)$.

\begin{tabular}{lccccrrr}
\hline Groups & No. & T0 & T1 & T2 & T3 & T4 & P-value \\
\hline I & 15 & $109.3 \pm 29.9$ & $118.0 \pm 37.8$ & $132.0 \pm 33.4$ & $140.0 \pm 56.3$ & $139.3 \pm 27.6$ \\
II & 14 & $103.3 \pm 19.2$ & $117.3 \pm 30.8$ & $146.0 \pm 22.3$ & $128.7 \pm 29.7$ & $106.0 \pm 29.0$ & $<0.12$ \\
III & 14 & $106.7 \pm 22.3$ & $103.3 \pm 20.9$ & $114.7 \pm 24.5$ & $130.0 \pm 29.8$ & $122.3 \pm 28.9$ & 0.04 \\
IV & 14 & $101.3 \pm 30.7$ & $94.7 \pm 25.3$ & $108.7 \pm 19.2$ & $117.3 \pm 25.5$ & $96.7 \pm 34.0$ \\
P-value & & 0.85 & 0.11 & $<0.01$ & 0.46 & 0.18 \\
\hline
\end{tabular}

Difference between groups by time period: T0, no significance. T1, I vs. IV, $\mathrm{P}=0.04$; and II vs. IV, $\mathrm{P}=0.04$. T2, I vs. IV, $\mathrm{P}=0.02$; II vs. III, $\mathrm{P}<0.01$; and II vs. IV, $\mathrm{P}=0.03$. T3, no significance. T4, I vs. II, $\mathrm{P}<0.01$; I vs. IV, $\mathrm{P}<0.01$; and III vs. IV, $\mathrm{P}=0.03$. Differences between time periods compared to T0 for each group: I, T0 vs. T3, $\mathrm{P}=0.03$; and T0 vs. T4, $\mathrm{P}=0.03$. II, T0 vs. T2, $\mathrm{P}<0.01$; and T0 vs. T3, $\mathrm{P}=0.01$. III, T0 vs. T3, $\mathrm{P}=0.02$. IV, no significance.

at T0 $(\mathrm{P}<0.05)$. The IL-17 levels in groups III and IV exhibited no significant differences during the entire period. The IL-17 levels in group IV at T2, T3 and T4 were significantly decreased compared to those in group I $(\mathrm{P}<0.05)$ (Table V).

\section{Discussion}

The stress induced by invasive surgery of the thoracic cavity may be associated with immunosuppression through a notable upregulation of immunoactive proinflammatory cytokines. Similar to other types of invasive surgery where extensive tissue damage is present, several major surgical operations have been previously associated with innate immune system dysfunction (6,7), although little has been done to address the poor effectiveness of modern analgesics in combatting this activity. In fact, activation of the neuroendocrine-immune axis following surgical procedures is an established hallmark of stress, tissue injury and infection. Notably, the secretion of glucocorticoids plays a major role in stress-induced suppression of immune-inflammatory reactions $(6,8-11)$, providing a potential target for future drug therapies. In order to develop more effective analgesic and anesthetic drugs, the complex interactions between stress hormones and the immune system following surgical stress, which is often responsible for evoking increased endogenous secretion of glucocorticoids and catecholamines, must be elucidated.
In order to highlight the effect of various drug treatments on this mechanism, increases in serum Cor, adrenaline and noradrenaline levels and changes in the levels of several associated interleukins may be measured, as in the technique adopted by the present study. Notably, IL- 6 is produced at the site of the surgical wound, where it subsequently enters systemic circulation and boosts serum IL-6 levels. Due to its previously described correlation with surgical severity and tissue injury, the levels of the proinflammatory cytokine IL-6 in the serum of surgical patients has been particularly useful in predicting the occurrence of hyperalgesia $(12,13)$. Although the pathways affecting IL-6 production and transport to the bloodstream have not been completely documented, the effect of serum IL-6 on the immune system is well documented for its effects on immunosuppression and pain modulation (14). Further investigations are required to determine the exact mechanism of IL- 6 transport into the bloodstream and the potential regulators of this pathway, which may serve as targets for future drug development.

The results of the present study support previous findings stating that tissue damage caused by invasive surgery increases plasma Cor and IL-6 concentrations, particularly in cases of surgery to the upper abdominal region, including partial gastrectomy or partial hepatectomy and esophageal surgery (15). Furthermore, the present study suggests that epidural blockade suppresses the stress-induced increase in Cor and IL-6 levels 
Table IV. Plasma concentrations of IFN- $\gamma$ and IL-4 (pg/ml) and IFN- $\gamma / \mathrm{IL}-4$ ratio.

A, IFN- $\gamma$ plasma concentration $(\mathrm{pg} / \mathrm{ml})$

\begin{tabular}{|c|c|c|c|c|c|c|c|}
\hline Groups & No. & T0 & $\mathrm{T} 1$ & $\mathrm{~T} 2$ & T3 & $\mathrm{T} 4$ & P-value \\
\hline I & 15 & $11.5 \pm 6.3$ & $11.4 \pm 3.4$ & $7.5 \pm 2.5$ & $5.3 \pm 3.5$ & $6.5 \pm 2.7$ & $<0.01$ \\
\hline II & 14 & $11.0 \pm 5.0$ & $11.2 \pm 3.2$ & $8.3 \pm 4.2$ & $7.3 \pm 2.6$ & $7.3 \pm 3.8$ & 0.01 \\
\hline III & 14 & $12.1 \pm 5.8$ & $11.8 \pm 5.3$ & $11.1 \pm 3.2$ & $8.3 \pm 2.3$ & $7.9 \pm 2.6$ & 0.02 \\
\hline IV & 14 & $12.0 \pm 4.3$ & $11.3 \pm 3.6$ & $11.2 \pm 5.6$ & $8.9 \pm 4.5$ & $8.6 \pm 3.1$ & 0.14 \\
\hline P-value & & 0.95 & 0.98 & 0.03 & 0.03 & 0.31 & \\
\hline
\end{tabular}

B, IL-4 plasma concentration $(\mathrm{pg} / \mathrm{ml})$

\begin{tabular}{|c|c|c|c|c|c|c|c|}
\hline Groups & No. & T0 & $\mathrm{T} 1$ & $\mathrm{~T} 2$ & $\mathrm{~T} 3$ & $\mathrm{~T} 4$ & P-value \\
\hline I & 15 & $5.2 \pm 1.2$ & $5.3 \pm 2.0$ & $7.0 \pm 2.0$ & $6.9 \pm 2.0$ & $7.7 \pm 2.3$ & $<0.01$ \\
\hline II & 14 & $5.2 \pm 2.0$ & $5.7 \pm 3.0$ & $7.0 \pm 3.0$ & $6.5 \pm 2.3$ & $6.5 \pm 2.1$ & 0.34 \\
\hline III & 14 & $5.4 \pm 1.7$ & $5.4 \pm 2.2$ & $5.5 \pm 2.7$ & $6.3 \pm 1.9$ & $6.0 \pm 2.5$ & 0.76 \\
\hline IV & 14 & $5.5 \pm 1.6$ & $5.4 \pm 2.0$ & $5.5 \pm 3.0$ & $6.3 \pm 3.0$ & $5.8 \pm 3.0$ & 0.89 \\
\hline P-value & & 0.95 & 0.96 & 0.23 & 0.34 & 0.18 & \\
\hline \multicolumn{8}{|c|}{$\mathrm{C}, \mathrm{IFN}-\gamma / \mathrm{IL}-4$ ratio } \\
\hline Groups & No. & T0 & $\mathrm{T} 1$ & $\mathrm{~T} 2$ & $\mathrm{~T} 3$ & $\mathrm{~T} 4$ & P-value \\
\hline I & 15 & $2.6 \pm 1.8$ & $2.5 \pm 1.0$ & $1.5 \pm 0.5$ & $1.0 \pm 0.8$ & $1.0 \pm 0.6$ & $<0.01$ \\
\hline II & 14 & $2.9 \pm 2.0$ & $2.7 \pm 1.0$ & $1.6 \pm 0.3$ & $1.4 \pm 0.7$ & $1.2 \pm 0.8$ & $<0.01$ \\
\hline III & 14 & $2.7 \pm 2.0$ & $2.6 \pm 0.8$ & $2.1 \pm 0.2$ & $1.2 \pm 0.8$ & $1.2 \pm 0.6$ & $<0.01$ \\
\hline IV & 14 & $2.8 \pm 1.7$ & $2.6 \pm 0.9$ & $2.2 \pm 0.3$ & $1.7 \pm 0.6$ & $1.9 \pm 0.7$ & 0.02 \\
\hline P-value & & 0.97 & 0.93 & $<0.01$ & 0.08 & 0.005 & \\
\hline
\end{tabular}

Differences in IFN- $\gamma$ between groups by time period: T0, no significance. T1, no significance. T2, I vs. III, P=0 .02; I vs. IV, P=0 .02. T3, I vs. III, $\mathrm{P}=0.02$; and I vs. IV, $\mathrm{P}<0.01$. T4, no significance. Differences in IFN- $\gamma$ between time periods compared to T0 for each group: I, T0 vs. T3, $\mathrm{P}<0.01$; and T0 vs. T4, $\mathrm{P}<0.01$. II, T0 vs. T3, $\mathrm{P}=0.01$; and T0 vs. T4, $\mathrm{P}=0.01$. III, T0 vs. T3, $\mathrm{P}=0.02$; and T0 vs. T4, $\mathrm{P}<0.01$. IV, no significance. Differences in IL-4 between groups by time period: T0, T1, T2, T3 and T4, no significance. Difference in IL-4 between time periods compared to T0 for each group: I, T0 vs. T2, P<0.01; T0 vs. T3, P=0.02; and T0 vs. T4, P<0.01. II, III and IV, no significance. Differences in IFN- $\gamma /$ IL-4 between groups by time period: T0, no significance. T1, no significance. T2, I vs. III, $\mathrm{P}<0.01$; I vs. IV, $\mathrm{P}<0.01$; II vs. III, $\mathrm{P}<0.01$; and II vs. IV, $\mathrm{P}<0.01$. T3, I vs. IV, $\mathrm{P}=0.01$. T4, I vs. IV, $\mathrm{P}<0.01$; II vs. IV, $\mathrm{P}<0.01$; and III vs. IV, $\mathrm{P}<0.01$. Differences in IFN- $\gamma /$ IL-4 between time periods compared to T0 for each group: I, T0 vs. T2, $\mathrm{P}<0.01$; T0 vs. T3, $\mathrm{P}<0.01$; and T0 vs. T4, $\mathrm{P}<0.01$. II, T0 vs. T2, $\mathrm{P}<0.01$; T0 vs. T3, P<0.01; and T0 vs. T4, $\mathrm{P}<0.01$. III, T0 vs. T3, $\mathrm{P}<0.01$; and T0 vs. T4, $\mathrm{P}<0.01$. IV, T0 vs. T3, $\mathrm{P}<0.01$; and T0 vs. T4, $\mathrm{P}=0.02$.

Table V. Plasma concentrations of IL-17 (ng/l).

\begin{tabular}{|c|c|c|c|c|c|c|c|}
\hline Groups & No. & T0 & $\mathrm{T} 1$ & $\mathrm{~T} 2$ & $\mathrm{~T} 3$ & $\mathrm{~T} 4$ & P-value \\
\hline I & 15 & $20.8 \pm 7.3$ & $26.5 \pm 6.7$ & $32.5 \pm 5.2$ & $33.3 \pm 8.2$ & $30.2 \pm 8.3$ & $<0.01$ \\
\hline II & 14 & $18.4 \pm 6.8$ & $23.3 \pm 8.2$ & $28.2 \pm 8.4$ & $26.5 \pm 9.9$ & $23.1 \pm 7.6$ & 0.03 \\
\hline III & 14 & $22.1 \pm 7.2$ & $23.1 \pm 7.2$ & $25.7 \pm 6.5$ & $26.2 \pm 7.5$ & $24.2 \pm 7.3$ & 0.52 \\
\hline IV & 14 & $19.6 \pm 8.6$ & $21.8 \pm 7.6$ & $22.4 \pm 7.3$ & $22.7 \pm 5.3$ & $21.3 \pm 6.2$ & 0.79 \\
\hline P-value & & 0.60 & 0.38 & $<0.01$ & $<0.01$ & 0.01 & \\
\hline
\end{tabular}

Differences in IL-17 between groups by time period: T0, no significance. T1, no significance. T2, I vs. III, $\mathrm{P}=0.01$; I vs. IV, $\mathrm{P}<0.01$; and II vs. IV, $\mathrm{P}=0.03$. T3, I vs. II, $\mathrm{P}=0.02$; I vs. III, $\mathrm{P}=0.02$; and I vs. IV, $\mathrm{P}<0.01$. T4, I vs. II, $\mathrm{P}=0.01$; I vs. III, $\mathrm{P}=0.03$; and I vs. IV, $\mathrm{P}<0.01$. Differences in IL-17 between time periods compared to T0 for each group: I, T0 vs. T1, $\mathrm{P}=0.03$; T0 vs. T2, $\mathrm{P}<0.01$; T0 vs. T3, $\mathrm{P}<0.01$; and T0 vs. T4, $\mathrm{P}<0.01$. II, T0 vs. T2, $\mathrm{P}<0.01$; and T0 vs. T3, $\mathrm{P}=0.01$. III and IV, no significance. 
subsequent to esophageal surgery, a finding which is likely to be consistent in other major surgeries of the upper abdominal region. This observation may be explained by the ability of the sympathetic nerve block induced by epidural anesthesia to reduce the surgical stress response, including reductions in plasma catecholamine and Cor levels, thus improving the overall immune response and limiting inflammation. Although there remains some controversy regarding the true value of sympathetic nerve blockade induced by epidural anesthesia in reducing surgical stress and the related immune response, an increasing number of researchers are reporting positive results. Upon application of epidural anesthesia, Tsui et al (16) observed fewer cardiovascular complications, a reduction in morbidity and mortality and a shorter duration of hospitalization for esophageal surgery patients treated with epidural analgesia, compared to patients treated with the traditional technique of controlled intravenous analgesia. Consistent with those results, the present study indicates that PCEA is more effective compared to PCIA for the inhibition of surgical stress and resultant immune activity, based on the plasma levels of certain indicative immune-related compounds.

In healthy mammals, including humans, Th1 and Th 2 cells interact to maintain the balance required for normal immunity. Imbalances in this ratio may result in moderate to severe inhibition or functional alteration to critical immune system activities. It was previously demonstrated that trauma and sepsis favor a Th2-dominant status $(17,18)$. As invasive surgeries often result in significant tissue trauma, the same conclusion may be drawn for surgical patients. This hypothesis is supported by the present findings of a Th2-dominant status in patients following esophageal surgery, which is considered to play a detrimental role in the immune response and may lead to increased neuropathic pain. General anesthesia combined with TEA or PCEA was shown to significantly inhibit the stress response and provide more effective postoperative pain relief, possibly by reducing the occurrence of complex neuropathic pain. In addition, this treatment was found to slow down the conversion of Th0 cells to Th2 cells, further contributing to maintaining the Th1/Th2 balance. Previously, anesthesia was shown to attenuate the suppression of immunity and preserve the Th1/Th2 cytokine balance subsequent to surgery (19). One of the predominant indicators of surgical stress is an increased Cor level, which is capable of affecting immune function in various tissues. Cor inhibits the differentiation of Th0 cells into Th1 cells, thus contributing to the development of a Th2-dominant status $(20,21)$.

By maintaining a BIS value within a range of 40-60 using intubation during surgery as a technique for anesthesia, the present study demonstrated that general anesthesia with TEA inhibited the onset of a Th2-dominant status and decreased plasma levels of Cor and IL-6 more efficiently compared to general anesthesia alone. Additionally, compared to PCIA, PCEA inhibited the Th2-dominant status more efficiently. TEA or PCEA were shown to be superior in attenuating the stress-induced adverse immune response associated with esophageal surgery. The present study also indicated that TEA or PCEA may be associated with reduced serum concentrations of Cor when compared to either general anesthesia and PCIA. TEA combined with PCEA may even facilitate the differentiation of Th0 cells into Th1 cells, inhibiting the Th2-dominant status and aiding in the depression of the stress-induced immune response.

Th17 cells secrete the pro-inflammatory cytokine IL-17, playing a role in inflammation and autoimmunity. In addition to IL-17, the inflammation-associated cytokines IL-6 and transforming growth factor- $\beta$ are also associated with Th17 cell differentiation from their native $\mathrm{CD} 4^{+}$cell predecessors, distinct from classical Th1 and Th2 cells. The Th17 cell response has been recently implicated in the response to several models of infection potentially associated with tissue trauma $(22,23)$, indicating a significance in postsurgical patients prone to infection. Evidence indicates that IL-17 is the major effector molecule produced by Th17 cells and that IL-17 stimulates several other cell types, such as endothelial cells, epithelial cells and macrophages, to produce numerous proinflammatory mediators, including IL-6 (24). The currently observed increase in plasma IL-17 concentrations during thoracic surgery, indicating a potential for attenuation by TEA or PCEA, is consistent with previous findings (25).

Although the stage of esophageal cancer, as well as other relevant pathological parameters, may be associated with inflammation and immune suppression following surgery, it is our hypothesis that the dominant role in immunosuppression and inflammation is caused by tissue damage during surgery. However, further investigation is required to determine which portion of the response is due to the pathological condition and which is due to surgical trauma. The present study conducted cytokine measurements over a 48 -h postoperative period, providing useful initial postsurgical results; however, our knowledge of long-term effects on stress and immune response is limited. During this measurement period, the cytokine levels remained elevated, although previous results suggest a return to baseline levels within approximately 1 week after surgery (26). Our present findings may also be limited by the inability to assess the scope of epidural blockade during the operation. The epidural blockade to the T4 dermatome was verified prior to induction of anesthesia and postoperatively; however, there remains a possibility that the epidural sympathetic blockade was inadequate during the operation or the postoperative period in groups II or IV. Furthermore, the propofol used may have also exerted anti-inflammatory effects capable of confounding these results, although this is unlikely. In order to verify these results, a larger and more diverse patient cohort is required to minimize the risk of type 2 error. Based on these initially positive results and their high potential for significantly improving the surgical results, future studies should further investigate the validity, underlying mechanism and application of these findings.

Immunosuppression is likely the result of a combination of pathological factors induced by disease, such as esophageal carcinoma, and tissue trauma caused by surgical treatment. The innate immune system exhibited signs of suppression in the first 2 days following thoracic surgery. Compared to general anesthesia alone or PCIA, TEA and PCEA appear to preferably promote Th0 cells to differentiate into Th1 cells, thereby inhibiting surgical stress by maintaining the Th1/Th2 ratio. Hence, TEA and PCEA may serve as immunoprotective agents for surgical patients based on the observed ability of these anesthetic and analgesic techniques to affect the differentiation of human helper T-lymphocytes, mediate stress and 
immune response and improve shot-term pain management. These findings may also provide a theoretical basis for optimization of anesthetic methods through the development and clinical application of anesthetic and analgesic combinations that effectively relieve stress without detrimentally affecting immune function.

In conclusion, general anesthesia combined with TEA and sole administration of PCEA were found to inhibit the stress response and minimize immune dysfunction, generating most pronounced results upon combination TEA/PCEA treatment.

\section{Acknowledgements}

This study was supported by an Outstanding Medical Professional Award from the Suzhou City Government (Suzhou, China) (grant no. RC0802) and by the '333 project' of Jiangsu province (Jiangsu, China) (grant no. BRA2011047).

\section{References}

1. Munford RS and Pugin J: Normal responses to injury prevent systemic inflammation and can be immunosuppressive. Am J Respir Crit Care Med 163: 316-321, 2001.

2. Watkins LR ang Maier SF: Beyond neurons: evidence that immune and glial cells contribute to pathological pain states. Physiol Rev 82: 981-1011, 2002.

3. Watkins LR and Maier SF: Implications of immune-to-brain communication for sickness and pain. Proc Natl Acad Sci USA 96: 7710-7713, 1999.

4. Afzali B, Mitchell P, Lechler RI, John S and Lombardi G: Translational mini-review series on Th17 cells: induction of interleukin-17 production by regulatory T cells. Clin Exp Immunol 159: 120-130, 2010.

5. Sarkar S, Cooney LA and Fox DA: The role of T helper type 17 cells in inflammatory arthritis. Clin Exp Immunol 159 225-237, 2010.

6. Duignan JP, Collins PB, Johnson AH and Bouchier-Hayes D: The association of impaired neutrophil chemotaxis with postoperative surgical sepsis. Br J Surg 73: 238-240, 1986.

7. Utoh J, Yamamoto T, Utsunomiya T, Kambara T, Goto H and Miyauchi Y: Effect of surgery on neutrophil functions, superoxide and leukotriene production. Br J Surg 75: 682-685, 1988.

8. Tonnesen $\mathrm{E}$ and Wahlgreen C: Influence of extradural and general anesthesia on natural killer cell activity and lymphocyte subpopulations in patients undergoing hysterectomy. Br J Anaesth 60: 500-507, 1988 .

9. Tonnesen E, Huttel MS, Christensen NJ and Schmitz O: Natural killer cell activity in patients undergoing upper abdominal surgery: relationship to the endocrine stress response. Acta Anaesthesiol Scand 28: 654-660, 1984

10. Kawasaki T, Ogata M, Kawasaki C, Tomihisa T, Okamoto K and Shigematsu A: Surgical stress induces endotoxin hyporesponsiveness and an early decrease of monocyte mCD14 and HLA-DR expression during surgery. Anesth Analg 92: 1322-1326, 2001.
11. Wakefield CH, Carey PD, Foulds S, Monson JR and Guillou PJ: Changes in major histocompatibility complex class II expression in monocytes and $\mathrm{T}$ cells of patients developing infection after surgery. Br J Surg 80: 205-209, 1993.

12. Naito Y, Tamai S, Shingu K, et al: Responses of plasma adrenocorticotropic hormone, cortisol, and cytokines during and after upper abdominal surgery. Anesthesiology 77: 426-431, 1992.

13. Holzheimer RG and Steinmetz W: Local and systemic concentrations of pro- and anti-inflammatory cytokines in human wounds. Eur J Med Res 5: 347-355, 2000.

14. De Jongh RF, Vissers KC, Meert TF, Booij LH, De Deyne CS and Heylen RJ: The role of interleukin-6 in nociception and pain. Anesth Analg 96: 1096-1103, 2003.

15. Cruickshank AM, Fraser WD, Burns HJ, Van Damme J and Shenkin A: Response of serum interleukin- 6 in patients undergoing elective surgery of varying severity. Clin Sci (Lond) 79: 161-165, 1990.

16. Tsui SL, Law S, Fok M, et al: Postoperative analgesia reduces mortality and morbidity after esophagectomy. Am J Surg 173: 472-478, 1997.

17. Murphey ED, Herndon DN and Sherwood ER: Gamma interferon does not enhance clearance of Pseudomonas aeruginosa but does amplify a proinflammatory response in a murine model of postseptic immunosuppression. Infect Immun 72: 6892-6901, 2004.

18. King MR, Ismail AS, Davis LS and Karp DR: Oxidative stress promotes polarization of human $\mathrm{T}$ cell differentiation toward a T helper 2 phenotype. J Immunol 176: 2765-2772, 2006.

19. Wada H, Seki S, Takahashi T, et al: Combined spinal and general anesthesia attenuates liver metastasis by preserving TH1/TH2 cytokine balance. Anesthesiology 106: 499-506, 2007.

20. Hertel VG, Olthoff D, Vetter B, Giessner O and Lange S: Comparison of various methods of anesthesia by plasma catecholamine determination. Anaesthesiol Reanim 20: 116-125, 1995.

21. Pirttikangas CO, Salo M, Mansikka M, Gronroos J, Pulkki K and Peltola O: The influence of anaesthetic technique upon the immune response to hysterectomy. A comparison of propofol infusion and isoflurane. Anesthesia 50: 1056-1061, 1995.

22. Oppeltz RF, Zhang Q, Rani M, Sasaki JR and Schwacha MG: Increased expression of cardiac IL-17 after burn. J Inflamm (Lond) 7: 38, 2010.

23. Jia S, Li C, Wang G, Yang J and Zu Y: The Thelper type 17/regulatory $\mathrm{T}$ cell imbalance in patients with acute Kawasaki disease. Clin Exp Immunol 162: 131-137, 2010.

24. Afzali B, Lombardi G, Lechler RI and Lord GM: The role of T helper 17 (Th17) and regulatory T cells (Treg) in human organ transplantation and autoimmune disease. Clin Exp Immunol 148: 32-46, 2007.

25. Fant F, Tina E, Sandblom D, et al: Thoracic epidural analgesia inhibits the neuro-hormonal but not the acute inflammatory stress response after radical retropubic prostatectomy. $\mathrm{Br}$ J Anaesth 110: 747-757, 2013.

26. Scheingraber S, Dobbert D, Schmiedel P, Seliger E and Dralle H: Gender-specific differences in sex hormones and cytokines in patients undergoing major abdominal surgery. Surg Today 35: 846-854, 2005. 